\title{
Direct Extraction of Normal Mapped Meshes from Volume Data
}

\author{
Mark Barry and Zoë Wood \\ California Polytechnic State University
}
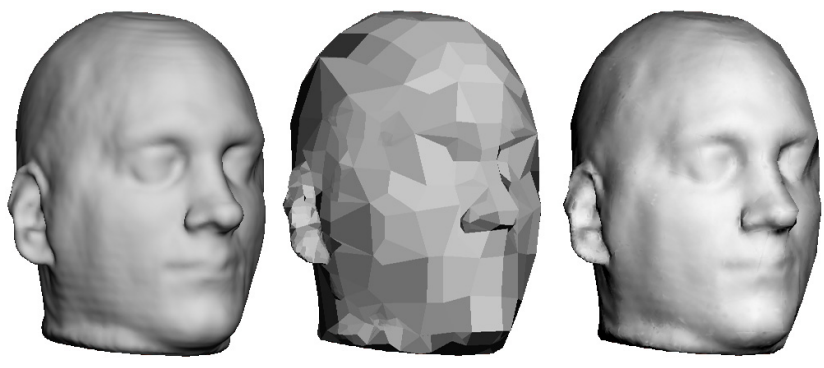

Figure 1. A human head extracted from a 130 x $128 \times 128$ volume. All meshes are dual contour surfaces. Left: 56,637 quads. Center: 1,406 quads. Right: same as center but with normal maps, generated with our algorithm in about 1 second.

\begin{abstract}
We describe a method of directly extracting a simplified contour surface along with detailed normal maps from volume data in one fast and integrated process. A robust dual contouring algorithm is used for efficiently extracting a high-quality "crack-free" simplified surface from volume data. As each polygon is generated, the normal map is simultaneously generated. An underlying octree data structure reduces the search space required for high to low resolution surface normal mapping. The process yields simplified meshes fitted with normal maps that accurately resemble their complex equivalents in one second on average.
\end{abstract}

\section{Introduction}

Volumetric data is a fundamental data structure having the benefit that it can describe surfaces, spatial extents and interior structures. In medical imaging, three-dimensional samplings of human body tissue obtained through magnetic resonance (MR) or computed tomography (CT) scans are captured as volume data. Volume data has also been used in the process of scanning physical objects to electronically capture their shape [7]. A popular method for viewing the object or structure that volume data describes requires extracting a contour surface or isosurface. Typically an extracted contour surface of high detail will contain a very large number of polygons. Though a highly detailed contour surface is often desirable, a high cost is incurred in terms of its storage space and rendering performance. This is why approximating the original mesh by reducing the extracted surface's size and complexity is common.

Ultimately, it is desirable to achieve the benefits of both worlds: the appearance of fine detail on the contour coupled with high performance. A technique known as normal mapping is one way to achieve this goal. Normal mapping "paints on" the fine details that are otherwise not captured on a low resolution surface. We present a new method for quickly generating simplified contour surfaces with high resolution detail using normal maps directly extracted from volume data. Current normal mapping algorithms are applicable to volume data only after a surface has been extracted, requiring a lengthy, multi-step process. We demonstrate a novel method of greatly shortcutting the current process by directly extracting a simplified surface along with normal maps in one integrated process, using the volume data structure to our advantage. The process is very fast - capable of extracting normal mapped meshes of various resolutions in the time of one second on average, giving the user the ability to quickly choose a mesh at the desired level of detail. The low resolution mesh extracted using our algorithm can render almost fourteen times faster then their high resolution equivalents. Yet even normal mapped low resolution meshes, with $92 \%$ fewer polygons, appear nearly identical to their high resolution equivalents (see Figure 6).

\section{Previous Work}

\subsection{Isosurface Extraction}

The Marching Cubes (MC) algorithm [16] "marches" through each cube of a uniform grid and generates polygons for each portion of the contour that intersects that cube. The MC algorithm can generate a high-quality mesh that captures a contour's fine details, but commonly generates very large meshes, ranging in the millions of polygons. Di- 


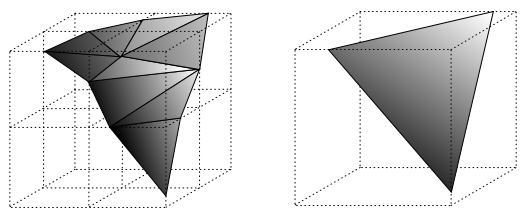

Figure 2. Left: a contour surface intersecting eight neighboring cubes (octant), generating nine triangles. Right: the collapsed cube approximation, generating one triangle.

rectly simplifying the volume through combining neighboring cubes will generate a simplified output mesh, but with the undesirable side effect that correct surface topology may not be preserved (see Figure 2). The Extended Marching Cubes algorithm [14] presents an improvement over the MC algorithm by generating additional contour vertices within cubes, which results in higher-quality meshes that capture features such as sharp edges. The downside of these methods is the generation of a high resolution mesh in order to display the contour surface's finest details.

\subsection{Adaptive Contouring}

Adaptive polygonization or adaptive contouring is one method of dealing with high resolution meshes produced by MC. An octree structure [17] [22] [21] can be generated to adaptively represent the volume data. An octant of the volume may be collapsed into a single cube if the intersecting contour remains accurately preserved. Collapsing cubes may be performed recursively on all branches of the octree, adapting to the complexity of the contour being extracted. The challenge with this method of adaptive contouring on simplified octrees is that the resulting polygonal mesh is not "water-tight". The problem of generating a closed polygonal mesh from a simplified octree has been extensively studied [4] [8] [22]. In general the solutions proposed are not perfect or require certain restrictions on how the octree is simplified.

\subsection{Dual Contouring}

Ju et. al. [13] present an alternative to previous adaptive contouring methods using dual contouring. Dual contouring methods generate a contour vertex within a cube's interior. The algorithm presented by $\mathrm{Ju}$ et. al. is an adaptive dual contouring method that produces a "crack-free" contour surface from a simplified octree. Unlike other adaptive contouring methods, this dual contouring method does not impose restrictions on how an octree is simplified nor requires any sort of crack patching. In addition, it performs as well as [14] in terms of preserving distinct features such as sharp edges.
In order to adaptively simplify the octree data structure that represents the volume data, the authors use the quadric error functions (QEFs) introduced in [9]. Associated with each leaf cube in the octree is a QEF that describes both the optimal placement of a contour vertex within the cube and the error quantifying how well that vertex approximates the contour surface. An octant may be collapsed if the resulting sum of QEFs yields an error less than a given tolerance. An output mesh is generated by connecting the contour vertices to form polygons. The algorithm produces either quads or triangles depending on the adaptive structure of the octree. The algorithm includes the option of restricting octant collapses to maintain surface topology through various levels of surface detail. Fixes and improvements to [13] are discussed in [23], [20], and [12]. Due to its ability to directly extract adaptive high quality meshes from volume data, we use this algorithm to create our low resolution meshes.

\subsection{Mesh Simplification and Normal Maps}

A popular approach to dealing with high resolution output meshes from MC involves first extracting a high resolution surface and later simplifying it as necessary [10] [9] [11]. These approaches are useful for creating lower resolution models, which conform well to the original geometry. In contrast, we are interested in methods that directly extract low resolution models from the volume data that conform well to the original mesh and more closely approximate the original meshes' high resolution appearance. One common approach to achieve the appearance of a high resolution mesh using simplified geometry is through the use of a normal map. A normal map [15] [6] "paints" a planar polygon, with varying normals. Illuminating the surface for rendering reveals not a uniformly shaded surface, but one with varying shading, making it visually convincing that the surface is not flat, but textured with fine details.

In [5], the authors describe a method by which the details from a high resolution mesh can be mapped to a low resolution approximation. Data such as texture values or surface normals found on the high resolution mesh are captured in texture maps (or normal maps) and applied to the low resolution approximation. The process first assumes that a high resolution mesh has been simplified into a low resolution approximation. For each polygon in the low resolution mesh, a texture map is created and sampled. For each sample, a ray is cast to determine the nearest corresponding point on the high resolution mesh. This is a costly operation that can involve searching every polygon in the high resolution mesh for a possible intersection with the ray. The authors of [5] optimize the search by partitioning the search space into cells. Note that creating this spatial partitioning data structure essentially requires the re-creation of a vol- 
ume data structure if the mesh came from a volume.

In contrast, our method avoids the initial step of requiring the extraction of a high resolution mesh. By using adaptive contouring, the algorithm is designed to directly extract the final simplified mesh. Another advantage to using our algorithm is a limited search space for mapping fine to coarse features, which is naturally implemented using the existing octree data structure. Collecting normals to create a normal map is limited to "searching" only four octree cubes that a polygon spans (see Section 3.2).

\subsection{Texture Mapping Progressive Meshes}

The authors of [19] present a method of constructing a progressive mesh such that all meshes in the progressive mesh sequence share a common texture parameterization. In the case of using a common normal map, all meshes at all resolutions retain the same fine details found on the highest-resolution mesh. In much the same way, our work allows the extraction of meshes at various resolutions that use normal maps to retain the same fine details found on a high resolution equivalent. The main difference is that the progressive mesh approach uses a single normal map for all mesh resolutions whereas our work generates a new set of normal maps for each mesh extracted. The single parameterization approach may be more efficient in the end but takes a substantially longer time to construct for all mesh resolutions. Our algorithm instead allows one to immediately switch between varying resolutions very quickly, thus avoiding any lengthy initial construction process.

\section{Algorithm Overview and Contributions}

We present a method to directly extract geometrically simplified, low polygon meshes with their accompanying normal maps from volume data. The algorithm follows these simple stages:

1. Create an adaptive octree data structure from the original volume using QEF.

2. Extract the dual contour using [13].

3. For each low resolution polygon, create the associated normal map using the octree data structure.

Steps one and two follow the work of [13] exactly and thus are not presented here in any detail. Step three is our main contribution as we know of no other method to directly extract normal mapped meshes from volume data. Not only is our method novel in its application to the volume domain, but our method of generating normal maps by gathering the fine data in a fine-to-coarse approach (versus a coarse-tofine approach) is likewise new (see Section 3.3). We discuss the details of the algorithm in the following sections.
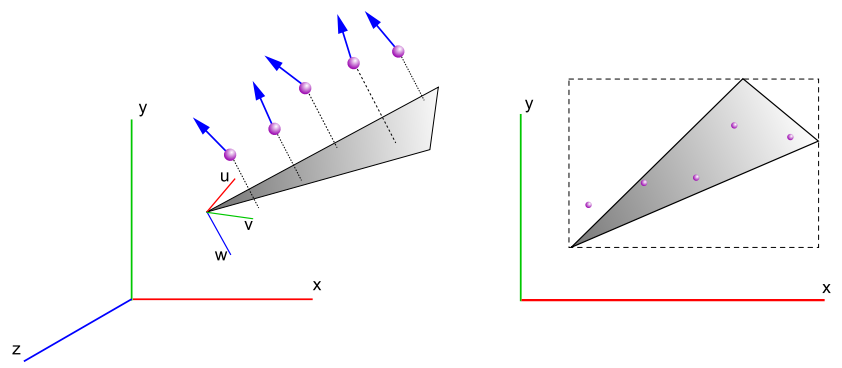

\section{Figure 3. Projection of fine-level contour ver- tices and their associated normals onto a contour triangle. The dotted bounding box on the right represents the normal map.}

\subsection{Dual Contouring of Volume Data With Normal Map Extraction}

Our goal is to extract a geometrically simplified surface with a normal map directly from volume data. In addition to being a robust adaptive contouring algorithm, dual contouring is ideally structured for easy extraction of normal maps as well. Thus we use the dual contouring method presented in [13] for both the creation of the octree using QEF and the surface extraction. Dual contouring builds the final mesh by connecting four minimizing vertices found within four neighboring cubes in the octree. If the adaptive structure of the octree includes neighboring cubes of different levels in the hierarchy, triangles are generated. The implementation treats triangles as quads having two vertices fused together. We therefore refer to all surface polygons as quads. To make up for the course appearance of the mesh alone, normal maps are created and applied to polygons as they are generated.

\subsection{Generating Normal Maps}

A normal map can be generated and stored in many different polygonal formats; triangles, rectangles, packed charts, etc. We choose to generate a normal map per quad. Normal map generation starts with the creation of a rectangular map. The dimensions of the rectangle are calculated based on the size of the quad. Because the quad is defined in three-dimensional space and the normal map is only twodimensional, the quad must be projected onto a two dimensional space.

The quad's orthonormal basis is computed and used to calculate the projection (see Figure 3). The orthonormal basis matrix is composed of three mutually perpendicular row vectors: $\vec{u}, \vec{v}$, and $\vec{w}$. The quad's normal is used for $\vec{w}$ and the vectors $\vec{u}$ and $\vec{v}$ must be computed. There are no constraints on the orientation of $\vec{u}$ nor $\vec{v}$, as long as all three vectors are mutually perpendicular. Choosing values for $\vec{u}$ and $\vec{v}$ translates into how the quad will be oriented on the 
normal map and determines the normal map's dimensions required to bound the quad. For most efficient use of space, the $\vec{u}$ vector can be oriented parallel with the quad's longest edge. The $\vec{v}$ vector can then be computed from $\vec{w} \times \vec{u}$. Note that for our implementation, we use the cross product of the quad's diagonals to compute $\vec{w}$.

The $x-y-z$ coordinate of each of the quad's vertices is multiplied with the basis matrix to perform the projection. After the projection, the z-component of the quad's vertices can be dropped and the coordinates treated as twodimensional. The two-dimensional quad is bounded with a rectangle representing the normal map. Next the normal map is filled, pixel-by-pixel, with the appropriate normal vector values. Sampling is chosen to match the sampling rate of the volume data.

The normal maps act to fill in the fine detail that the simplified contour surface lacks. The finest detail that can be obtained comes from the contour vertices generated at the finest levels ("leaves") of the octree. Thus the process of generating a normal map involves capturing the normals from these fine-level contour vertices. In general, normals for a low resolution quad are obtained by collecting all the fine-level contour vertices contained within four spanning cubes in the octree. Recall that contour vertices are contained within an octree cube and surface polygons are formed by connecting four minimizing vertices, thus each polygon spans four octree cubes. Each cube calls a recursive function for each of its child cubes, traversing down the octree of sub-cubes until reaching a leaf cube. At a leaf cube, contour vertices, if any, are extracted and returned in an array. As all calls to the recursive function return, the arrays of fine-level contour vertices are combined. These fine-level contour vertices are then projected onto the quad's normal map.

\subsection{Interpolating Scattered Normals}

The projection step scatters "normal-defined" pixels across the normal map but still leaves the remaining pixels' normal values undefined. In order to completely fill the normal map, the normal values at the projected points must be interpolated across undefined pixels. The fact that these points are spaced in a random fashion makes interpolation a nontrivial problem. Many methods of interpolating scattered data are available [1] but a Delaunay Triangulation approach was initially implemented. The Delaunay Triangulation interconnects a set of scattered points and produces a two-dimensional triangle mesh. It tends to maximize the minimum angle of all the triangles' angles in the triangulation, avoiding any "sliver" triangles. To interpolate normals among points, pixels within each Delaunay triangle are filled according to the Phong shading model [18].

Interpolating projected vertices using the Delaunay Tri- angulation results in a visually acceptable interpolation but the process is fairly slow. Experimentation led to the discovery of a fast approximation that yields equally good results. This approximation works by first initializing the entire normal map with the contour quad's normal. Taking no further steps in modifying the normal maps, the resulting normal mapped mesh would appear identical to a flatshaded mesh without normal maps. After initialization, the fine-level vertices are projected onto the normal map as before, overwriting any initialized pixels. All normal mapped meshes displayed in the figures use this method. Other interpolation methods could certainly be explored in future work, however, our simplified approximation works surprisingly well.

Note that most previous work [5] [19] for generating normal maps use a ray shooting technique, where texel samples on the coarse polygon are sampled by searching for an associated point on the high resolution mesh. In this way, these approaches generate a densely sampled normal map for the coarse mesh, where many of the sampled normals will be an interpolated normal from an interior point on the face of the fine mesh. We take the opposite approach by projecting the high resolution sample points to the coarse mesh and then interpolating the data. This approach is arguably faster, not only because of the octree data structure but because of the number of samples being mapped from fine to coarse (versus the many texel samples generated across the coarse face needing an associated mapping to the fine mesh). Our approach could be considered as less accurate because we use less samples from the fine mesh, however, the only sample points that are lost are the interpolated normals from the interiors of the high resolution faces. We found that our fineto-coarse approach performed not only very quickly but also with visually pleasing results.

\subsection{Algorithm Summary}

The following outlines a summary of the final algorithm for extracting normal maps from volume data using dual contouring.

1. Convert raw volume data to octree structure. This is a one-time step performed only if needed.

2. Simplify octree using QEF.

3. Extract contour surface and normal maps.

(a) Use the dual contouring algorithm to extract quads.

(b) For each quad created, generate its normal map.

i. Calculate the quad's orthonormal basis. 
ii. Transform the quad's vertices into twodimensional space by using the quad's orthonormal basis.

iii. Collect all fine-level contour vertices for the quad.

iv. Project all fine-level contour vertices onto the quad.

v. Bound the projected quad with a rectangular normal map.

A. Calculate the height and width of the normal map. Use the same sampling rate used for the volume space, where one unit equals the length of a finestlevel cube.

B. Shift the quad, normal map, and all projected contour vertices so that the normal map's $\mathrm{x}$ and $\mathrm{y}$ coordinates are positive.

vi. Interpolate among the projected contour vertices to fill undefined normal map pixels.

For complete details on the algorithm, please see [2].

\subsection{Extracting Normal Maps From Im- plicit Surfaces}

In the process of developing this work we also explored extracting normal maps and simplified meshes for implicit surfaces [3]. We present our results here as we know of no other previous work for generating normal mapped isosurfaces from implicit equations. To extract the implicit surface mesh, the standard MC algorithm is used without incorporating any adaptive contouring techniques. First, a section of three-dimensional space is blocked off and subdivided into a uniform array of cubes. For each of the cubes' corner vertices, the three-dimensional $x-y-z$ coordinates are plugged into the implicit equation to calculate its scalar value. To produce a visually accurate model of the implicit surface without using normal maps, the threedimensional space must be divided into smaller cubes so that a finer sampling is possible. But because the final goal is to let the normal maps capture the fine detail rather than the mesh's underlying geometry, the space is subdivide into relatively large cubes. This results in a polygonalized implicit surface that appears rather rough - only approximating the general shape of the surface (see Figure 4 center).

Normal maps are generated exactly the same as with the discrete volume with the exception that normals are computed by plugging the three-dimensional $\mathrm{x}-\mathrm{y}-\mathrm{z}$ coordinates associated with each normal map pixel into the equation describing the implicit equation's gradient. Because the gradient is defined continuously everywhere, it is very straight
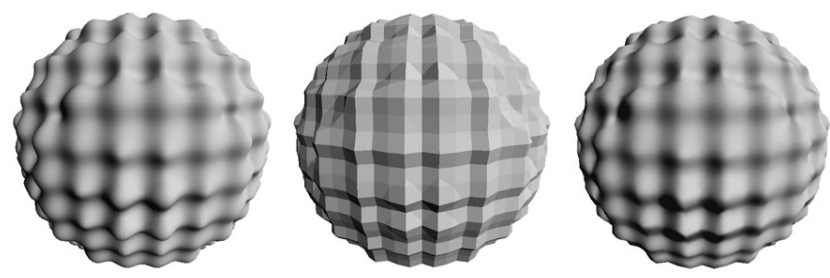

Figure 4. Left: high resolution implicit surface with 138,632 triangles. Center: simplified flat-shaded surface with 8,216 triangles. Right: same geometry as the center but with normal maps applied.

forward to determine normals for all pixels in the normal map. Though the process of generating normal maps for implicit surfaces works very nicely (see Figure 4), our work is focused on the more general domain of discrete volume data.

\section{Results}

One important measure of success is how well the low resolution normal mapped surface resembles the original high resolution surface without normal maps. Figures 1, 5 9 compare a low resolution and normal mapped mesh to its high resolution equivalent, demonstrating the excellent results from using our algorithm. Another factor to consider is the speed at which a mesh, along with its normal maps, can be extracted from the volume. The longest extraction time for our algorithm was for the dragon mesh from a $356 \times 161 \times 251$ volume, along with normal maps, at the highest resolution. This operation takes a little over $3 \mathrm{sec}-$ onds. Extracting simpler meshes (which would be the more frequent request) takes less time - an average of 1 second for the meshes shown in the following figures. This speed makes it easy for a user to quickly switch between varying levels of mesh resolution. A user may wish to begin with a low resolution mesh for fast display and cursory examination. Then as the user desires more geometric accuracy, a higher resolution mesh may be quickly extracted. It is very important to emphasize that a low resolution mesh is directly extracted. The process is not a matter of taking a high resolution mesh and simplifying it.

A fast rendering time is one of the main motivations for using normal mapped surfaces. Table 1 compares the rendering performance of high resolution meshes to low resolution, normal mapped, meshes. It is clear that rendering low resolution normal mapped meshes is a lot faster, yet they retain a significant amount of detail as shown in Figures 1,5 - 9 .

For the dragon example, even on the lowest resolution meshes, the dragon's scales are still clearly defined. The 
flat-shaded meshes alone do not come near to displaying that kind of detail. At the lowest resolutions, maintaining correct topology of the original high resolution mesh begins to fail. But even as the quality of the mesh breaks down, the applied normal maps still pick up the fine details. In the extreme example of Figure 7 (Right), the flat-shaded mesh alone hardly resembles the dragon model. But even with the poor underlying mesh geometry, applying normal maps immediately restores most of the finer details.

Figures 1, 8 - 9 demonstrate that the normal map extraction process works beautifully for a number of different volume data sets. In particular, figures of the mouse embryo and human head demonstrate that the process works equally well for medical imaging applications.

\section{Conclusion and Future Work}

We describe a method of directly extracting normal mapped contour surfaces from volume data. This method greatly shortcuts the current multi-step process of extracting a high resolution mesh, simplifying it, and generating normal maps. Instead, a high resolution mesh is never extracted. A low resolution mesh is extracted directly from the volume data, with normal maps immediately generated and applied. The process of generating normal maps is greatly streamlined due to the use of an octree data structure and the dual contouring algorithm. The visual results of this process are of excellent quality - the low resolution normal mapped meshes closely resemble their high resolution equivalents. This process is also very fast - generating and displaying a normal mapped surface in less than a few seconds.

Though we describe a method that is fast and yields excellent visual results, there is always room for improvement. One inefficiency in the system as it stands is that each polygon allocates its own normal map. In addition, the space efficiency is very poor. Ideally the collection of normal maps could be packed into one or a few atlases [5] [19]. Though the result would be a more efficient use of memory space, the packing process is computationally intensive.

Another optimization relates to collecting normals into the normal map. Since more than one quad may intersect a single cube, the process of collecting the fine-level vertices is performed more than once. This is an inefficiency that may be remedied by memoizing the collection of fine-level vertices within each cube.

Though we use dual contouring's QEF metric for mesh simplification, the use of other error metrics could be explored. The QEF metric aims to preserve the most prominent features such as sharp edges. Perhaps preserving certain sharp edges would not be necessary if they were instead captured in a normal map. In addition, more complex interpolation methods for interpolating normal data could be explored.
Finally, our work could be extended for use in computer games. Destructible game objects could be implemented similar to the work presented in [13] by representing all objects as volumes and performing Constructive Solid Geometry (CSG) operations on them. Referencing the rendering times in Table 1, it would be virtually impossible to include any of the high resolution meshes in a real-time game. But it would certainly be reasonable to include the normal mapped equivalents. In some cases, depending upon the level of "destruction", existing geometry would not need to be modified at all, but instead only the normal maps.

\section{References}

[1] I. Amidror. Scattered data interpolation methods for electronic imaging systems: a survey. Journal of Electronic Imaging, 11:157-176, April 2002.

[2] M. Barry. Direct extraction of normal maps from volume data. Technical Report CPSLO-CSC-07-01, California Polytechnic State University, 2007.

[3] J. Bloomenthal and B. Wyvill, editors. Introduction to Implicit Surfaces. Morgan Kaufmann Publishers Inc., San Francisco, CA, USA, 1997.

[4] P. Cignoni, F. Ganovelli, C. Montani, and R. Scopigno. Reconstruction of topologically correct and adaptive trilinear isosurfaces. Computers and Graphics, 24(3):399-418, 2000.

[5] P. Cignoni, C. Montani, R. Scopigno, and C. Rocchini. A general method for preserving attribute values on simplified meshes. In VIS '98: Proceedings of the conference on Visualization '98, pages 59-66, Los Alamitos, CA, USA, 1998. IEEE Computer Society Press.

[6] J. Cohen, M. Olano, and D. Manocha. Appearancepreserving simplification. In SIGGRAPH '98: Proceedings of the 25th annual conference on Computer graphics and interactive techniques, pages 115-122, New York, NY, USA, 1998. ACM Press.

[7] B. Curless and M. Levoy. A volumetric method for building complex models from range images. In SIGGRAPH '96: Proceedings of the 23rd annual conference on Computer graphics and interactive techniques, pages 303-312, New York, NY, USA, 1996. ACM Press.

[8] S. F. Frisken, R. N. Perry, A. P. Rockwood, and T. R. Jones. Adaptively sampled distance fields: a general representation of shape for computer graphics. In SIGGRAPH '00: Proceedings of the 27th annual conference on Computer graphics and interactive techniques, pages 249-254, New York, NY, USA, 2000. ACM Press/Addison-Wesley Publishing Co.

[9] M. Garland and P. S. Heckbert. Surface simplification using quadric error metrics. In SIGGRAPH '97: Proceedings of the 24th annual conference on Computer graphics and interactive techniques, pages 209-216, New York, NY, USA, 1997. ACM Press/Addison-Wesley Publishing Co.

[10] P. S. Heckbert and M. Garland. Survey of polygonal surface simplification algorithms. Technical report, 1997. 


\begin{tabular}{|c|c|c|c|c|c|c|}
\hline & \multicolumn{2}{|c|}{ Quad Count } & & \multicolumn{2}{|c|}{ Render Time (ms) } & \\
\hline \hline & Hi-Res & Lo-Res & Reduction & Hi-Res & Lo-Res & Speedup \\
\hline Figure 1 & 56,637 & 1,406 & $97.5 \%$ & 91 & 3 & 30.3 \\
\hline Figure 5 & 225,467 & 43,850 & $80.6 \%$ & 360 & 90 & 4.0 \\
\hline Figure 6 & 225,467 & 16,388 & $92.7 \%$ & 360 & 26 & 13.8 \\
\hline Figure 7 (L) & 225,467 & 558 & $99.8 \%$ & 360 & 1 & 360 \\
\hline Figure 7 (R) & 225,467 & 65 & $99.97 \%$ & 360 & 0.3 & 1200 \\
\hline Figure 8 & 150,823 & 10,950 & $92.7 \%$ & 245 & 22 & 11.1 \\
\hline Figure 9 & 64,896 & 3,035 & $95.3 \%$ & 103 & 6 & 17.2 \\
\hline
\end{tabular}

Table 1. Performance data for the examples shown in Figures 1, 5 - 9.

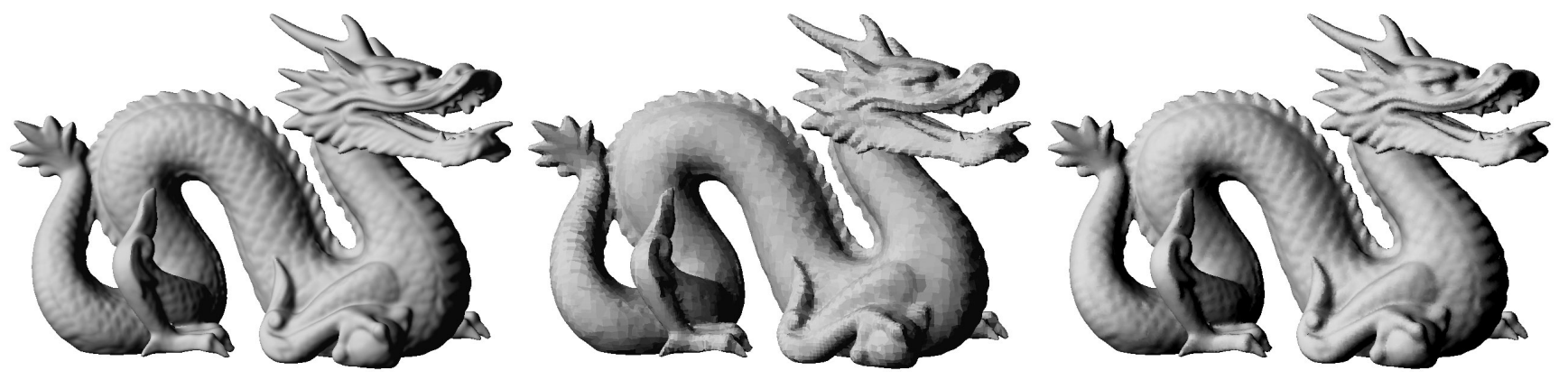

Figure 5. All dragon models extracted from a $356 \times 161 \times 251$ volume. All meshes are dual contour surfaces. Left: high resolution $-225,467$ quads. Center: low resolution $-43,850$ quads. Right: same as center but with normal maps applied.
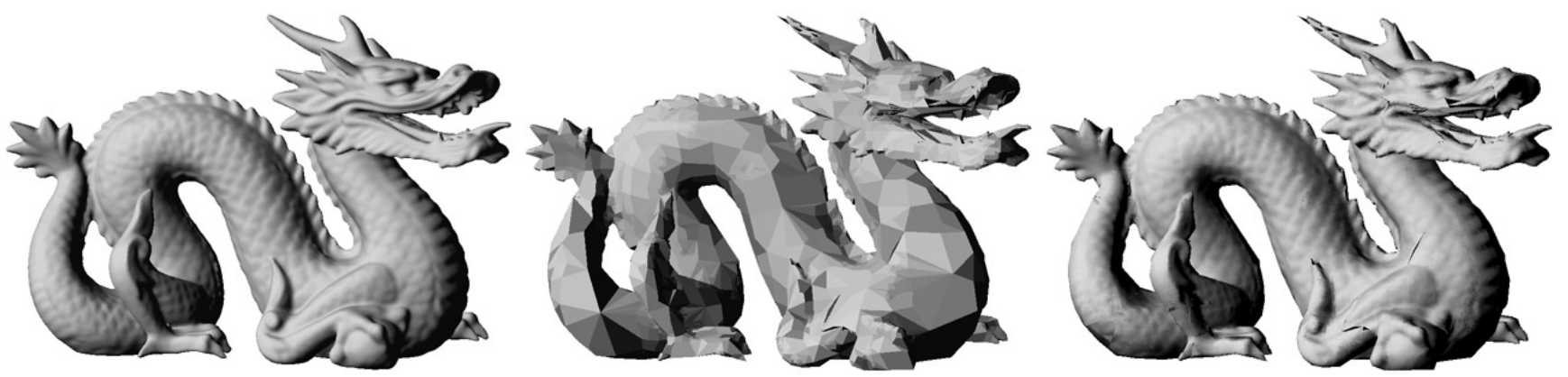

Figure 6. Left: 225,467 quads. Center: 16,388 quads. Right: same as center but with normal maps applied.
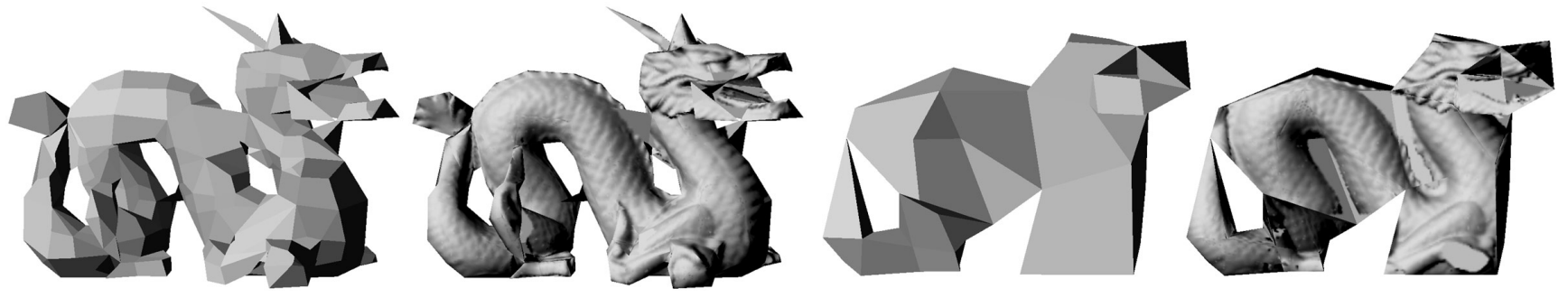

Figure 7. Left two: flat-shaded and normal mapped dual contour surface (558 quads). Right two: flat-shaded and normal mapped dual contour surface (65 quads). 

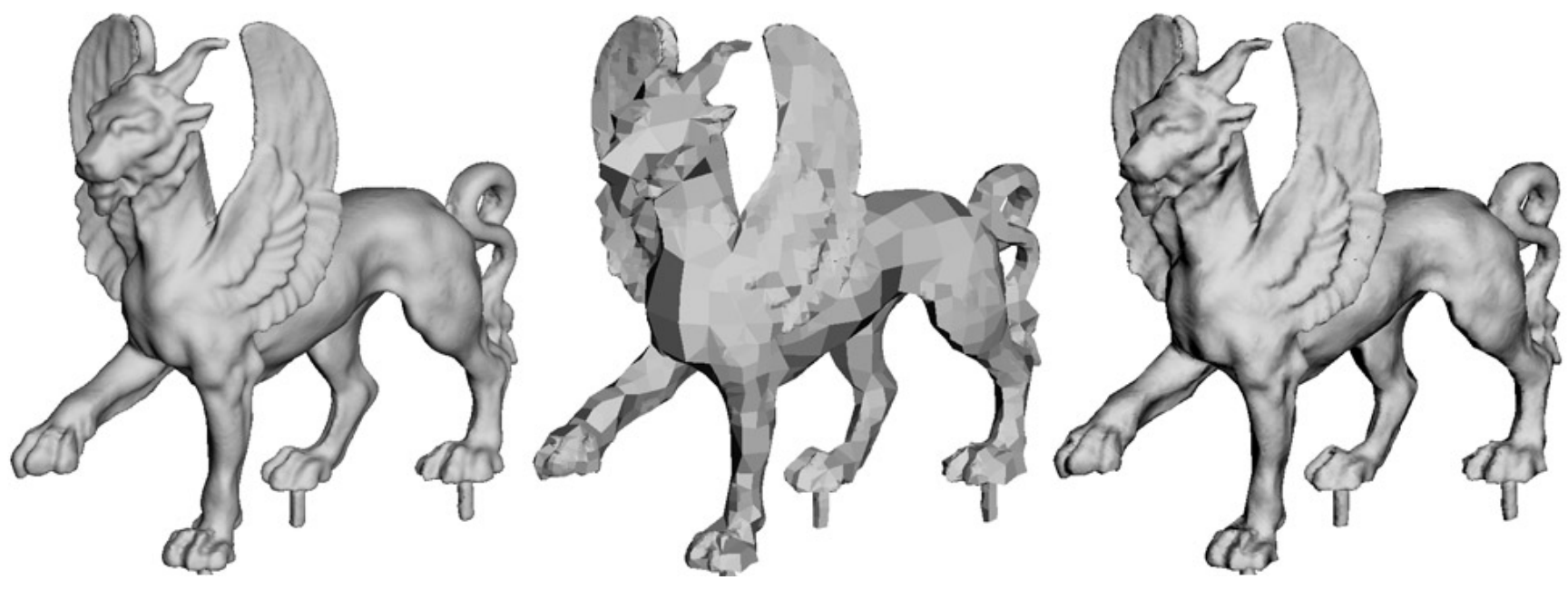

Figure 8. A mythical creature extracted from a $316 \times 148 \times 332$ volume. All meshes are dual contour surfaces. Left: 150,823 quads. Center: 10,950 quads. Right: same as center but with normal maps.
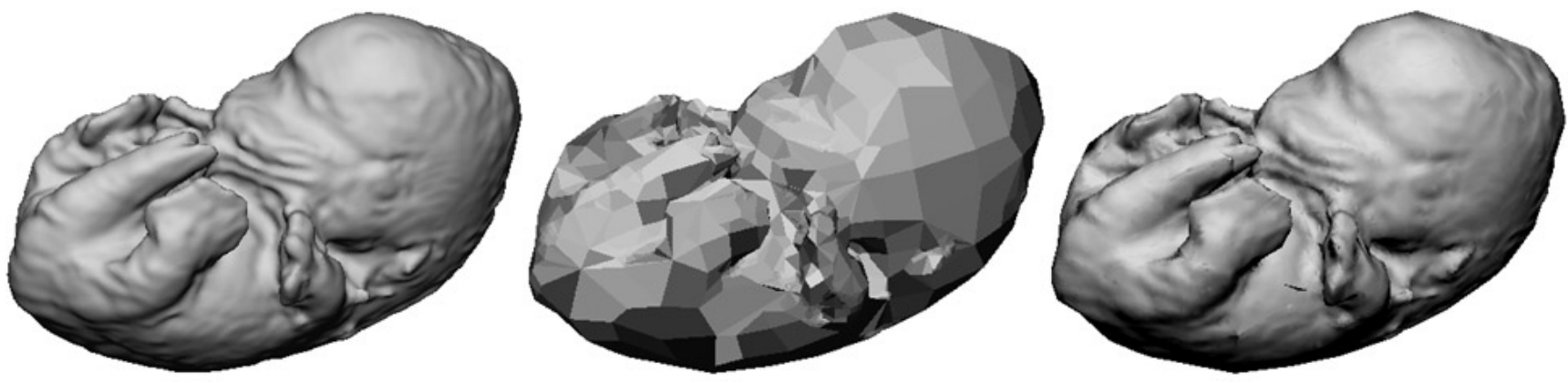

Figure 9. A mouse embryo extracted from a $256 \times 128 \times 128$ volume. All meshes are dual contour surfaces. Left: 64,896 quads. Center: 3,035 quads. Right: same as center but with normal maps.

[11] H. Hoppe. Progressive meshes. In SIGGRAPH '96: Proceedings of the 23rd annual conference on Computer graphics and interactive techniques, pages 99-108, New York, NY, USA, 1996. ACM Press.

[12] IEEE Computer Society. Intersection-free Contouring on An Octree Grid. IEEE, 2006.

[13] T. Ju, F. Losasso, S. Schaefer, and J. Warren. Dual contouring of hermite data. In SIGGRAPH '02: Proceedings of the 29th annual conference on Computer graphics and interactive techniques, pages 339-346, New York, NY, USA, 2002. ACM Press.

[14] L. P. Kobbelt, M. Botsch, U. Schwanecke, and H.-P. Seidel. Feature sensitive surface extraction from volume data. In SIGGRAPH '01: Proceedings of the 28th annual conference on Computer graphics and interactive techniques, pages 5766, New York, NY, USA, 2001. ACM Press.

[15] V. Krishnamurthy and M. Levoy. Fitting smooth surfaces to dense polygon meshes. In SIGGRAPH '96: Proceedings of the 23rd annual conference on Computer graphics and interactive techniques, pages 313-324, New York, NY, USA, 1996. ACM Press.

[16] W. E. Lorensen and H. E. Cline. Marching cubes: A high resolution 3d surface construction algorithm. In SIGGRAPH '87: Proceedings of the 14th annual conference on Computer graphics and interactive techniques, pages 163-169, New York, NY, USA, 1987. ACM Press.
[17] D. Meagher. Geometric modeling using octree encoding. In Computer Graphics and Image Processing, pages 129-147, 1982.

[18] B. T. Phong. Illumination for computer generated pictures. Commun. ACM, 18(6):311-317, 1975.

[19] P. V. Sander, J. Snyder, S. J. Gortler, and H. Hoppe. Texture mapping progressive meshes. In SIGGRAPH '01: Proceedings of the 28th annual conference on Computer graphics and interactive techniques, pages 409-416, New York, NY, USA, 2001. ACM Press.

[20] S. Schaefer, T. Ju, and J. Warren. Manifold dual contouring. IEEE Transactions on Visualization and Computer Graphics, 2007. to appear.

[21] R. Shekhar, E. Fayyad, R. Yagel, and J. F. Cornhill. Octreebased decimation of marching cubes surfaces. In VIS '96: Proceedings of the 7th conference on Visualization '96, pages 335-ff., Los Alamitos, CA, USA, 1996. IEEE Computer Society Press.

[22] J. Wilhelms and A. V. Gelder. Octrees for faster isosurface generation. ACM Trans. Graph., 11(3):201-227, 1992.

[23] N. Zhang, W. Hong, and A. Kaufman. Dual contouring with topology-preserving simplification using enhanced cell representation. In VIS '04: Proceedings of the conference on Visualization '04, pages 505-512, Washington, DC, USA, 2004. IEEE Computer Society. 